\title{
Comparison among Magnus/Floquet/Fer expansion schemes in solid-state NMR.
}

\author{
AUTHOR(S): \\ Takegoshi, K; Miyazawa, Norihiro; Sharma, \\ Kshama; Madhu, P K
}

\section{CITATION:}

Takegoshi, K ...[et al]. Comparison among Magnus/Floquet/Fer expansion schemes in solid-state NMR.. The Journal of chemical physics 2015, 142(13): 134201.

\section{ISSUE DATE: \\ 2015-04-02}

URL:

http://hdl.handle.net/2433/198276

\section{RIGHT:}

(c) 2015 American Institute of Physics. This article may be downloaded for personal use only. Any other use requires prior permission of the author and the American Institute of Physics. 


\section{A|P| $\begin{aligned} & \text { The Journal of } \\ & \text { Chemical Physics }\end{aligned}$}

Comparison among Magnus/Floquet/Fer expansion schemes in solid-state NMR

K. Takegoshi, Norihiro Miyazawa, Kshama Sharma, and P. K. Madhu

Citation: The Journal of Chemical Physics 142, 134201 (2015); doi: 10.1063/1.4916324

View online: http://dx.doi.org/10.1063/1.4916324

View Table of Contents: http://scitation.aip.org/content/aip/journal/jcp/142/13?ver=pdfcov

Published by the AIP Publishing

\section{Articles you may be interested in}

Introduction of the Floquet-Magnus expansion in solid-state nuclear magnetic resonance spectroscopy

J. Chem. Phys. 135, 044109 (2011); 10.1063/1.3610943

Solid-State NMR on Polymers under Mechanical Stress

AIP Conf. Proc. 1330, 109 (2011); 10.1063/1.3562245

Solid-state NMR covariance of homonuclear correlation spectra

J. Chem. Phys. 128, 134502 (2008); 10.1063/1.2884341

Accurate measurement of $\mathrm{C} 13-\mathrm{N} 15$ distances with solid-state NMR

J. Chem. Phys. 124, 144508 (2006); 10.1063/1.2191043

Nuclear Spin Polarizer for Solid-State NMR Quantum Computers

AIP Conf. Proc. 772, 1471 (2005); 10.1063/1.1994671

How can you REACH $100 \%$

of researchers at the Top 100

Physical Sciences Universities?

With The Journal of Chemical Physics.

AIP $\mid \begin{aligned} & \text { The Journal of } \\ & \text { Chemical Physics }\end{aligned}$

THERE'S POWER IN NUMBERS. Reach the world with AIP Publishing.

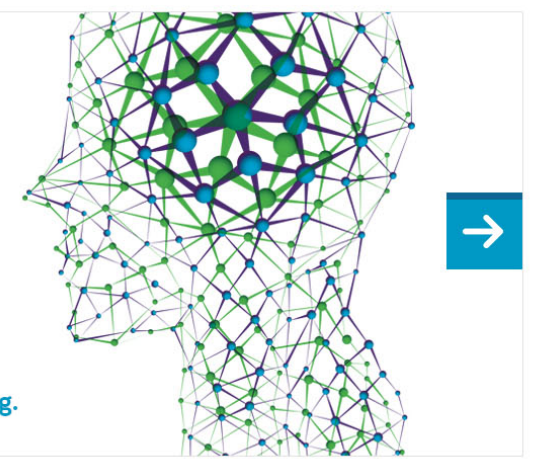




\title{
Comparison among Magnus/Floquet/Fer expansion schemes in solid-state NMR
}

\author{
K. Takegoshi, ${ }^{1, a)}$ Norihiro Miyazawa, ${ }^{1}$ Kshama Sharma, ${ }^{2}$ and P. K. Madhu ${ }^{2,3}$ \\ ${ }^{1}$ Division of Chemistry, Graduate School of Science, Kyoto University, 606-8502 Kyoto, Japan \\ ${ }^{2}$ TIFR Centre for Interdisciplinary Sciences, 21 Brundavan Colony, Narsingi, Hyderabad 500 075, India \\ ${ }^{3}$ Department of Chemical Sciences, Tata Institute of Fundamental Research, Homi Bhabha Road, Colaba, \\ Mumbai 400 005, India
}

(Received 4 January 2015; accepted 16 March 2015; published online 2 April 2015)

\begin{abstract}
We here revisit expansion schemes used in nuclear magnetic resonance (NMR) for the calculation of effective Hamiltonians and propagators, namely, Magnus, Floquet, and Fer expansions. While all the expansion schemes are powerful methods there are subtle differences among them. To understand the differences, we performed explicit calculation for heteronuclear dipolar decoupling, cross-polarization, and rotary-resonance experiments in solid-state NMR. As the propagator from the Fer expansion takes the form of a product of sub-propagators, it enables us to appreciate effects of time-evolution under Hamiltonians with different orders separately. While 0th-order average Hamiltonian is the same for the three expansion schemes with the three cases examined, there is a case that the 2nd-order term for the Magnus/Floquet expansion is different from that obtained with the Fer expansion. The difference arises due to the separation of the 0th-order term in the Fer expansion. The separation enables us to appreciate time-evolution under the 0th-order average Hamiltonian, however, for that purpose, we use a so-called left-running Fer expansion. Comparison between the left-running Fer expansion and the Magnus expansion indicates that the sign of the odd orders in Magnus may better be reversed if one would like to consider its effect in order. (C 2015 AIP Publishing LLC. [http://dx.doi.org/10.1063/1.4916324]
\end{abstract}

\section{INTRODUCTION}

In nuclear magnetic resonance (NMR), it is frequently necessary to examine effects of complex time-dependent spin interactions on NMR observables. The time dependence can arise from the time-dependent radiofrequency (RF) field and additionally from magic-angle spinning (MAS) in solid-state NMR. For a given time-dependent spin Hamiltonian $\mathcal{H}_{0}(t)$, the propagator is written as

$$
U(t)=T \exp \left(-i \int_{0}^{t} \mathcal{H}_{0}\left(t^{\prime}\right) d t^{\prime}\right)
$$

where $T$ is the Dyson time-ordering operator. In case of calculating evolution of spins under periodic perturbations, such as multiple pulse sequences and/or sample rotation, the average Hamiltonian theory allows us to calculate a time-independent effective Hamiltonian, which is given as a kind of "average" of interactions over a cycle time $\tau_{c}$ defined for a periodic Hamiltonian as $\mathcal{H}_{0}(t)=\mathcal{H}_{0}\left(t+\tau_{c}\right){ }^{1-3}$

The time-independent effective Hamiltonian (the average Hamiltonian) is generally expanded to an infinite series of $n$ thorder average Hamiltonians $(n=0,1, \ldots)$, whose magnitude decreases in order of $n$. So far, the scheme called as the Magnus expansion ${ }^{4}$ has been a working horse in the average Hamiltonian theory. For a system with its Hamiltonian being simply given by a Fourier series, the Floquet theory ${ }^{5}$ can be applied. In the framework of the Floquet theory, a so-called operatorbased Floquet theory has been developed, which gives conve-

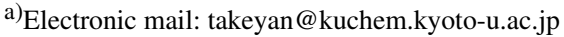

nient forms of $n$ th-order average Hamiltonians. ${ }^{6,7}$ Hereafter, we shall refer the expansion scheme based on the Floquet theory as the Floquet expansion. Recently, Madhu and Kurur adopted the Fer expansion ${ }^{8}$ and pointed out its several unique features by evaluating the Bloch-Siegert shift and continuouswave (CW) decoupling. ${ }^{9}$ In that work, it was claimed that the Fer expansion has several beneficial points. Further, it was stated that the effective Hamiltonian for a given situation as obtained in the Magnus expansion and the Fer expansion could be different. Mananga has applied the Fer expansion to examine the magic-echo sequence and showed that the Fer expansion is advantageous over the Magnus expansion in calculation of higher-order corrections. ${ }^{10}$

The most salient difference of the Fer expansion may be that it expands a propagator in the form of a product of propagators with $n$ th-order Hamiltonians as $U(t)$ $=\prod_{n=0}^{\infty} \exp \left\{-i t \mathcal{H}^{(n)}(t)\right\}$. This enables us to appreciate, for example, time-evolution governed by the 0th-order Hamiltonian separately from those by higher-order Hamiltonians. However, to examine this feature, one has to reverse the array of the Hamiltonians. For that purpose, we also examine a socalled left-running Fer expansion and compare the three expansion schemes (Magnus/Floquet/Fer) for three representative experiments, namely, $\mathrm{CW}$ decoupling, cross-polarization (CP), and rotary-resonance recoupling (R3) ${ }^{11}$ under sample spinning. CW decoupling is examined first as its 0th-order Hamiltonian is 0 , so that we can naively expect that the higher-order Hamiltonians are similar for the three expansion schemes. For $\mathrm{CP}$ and R3, their 0th-order Hamiltonians are not 0 , and we expect to find some subtle differences among the three expan- 
sion schemes. In fact for the R3 case, the 2nd-order average Hamiltonian obtained with the Magnus/Floquet expansion is different from that obtained by using the Fer expansion. We show that the latter Hamiltonian can also be derived approximately for the Magnus/Floquet expansion. It is concluded that the Fer expansion is more suitable for examination of solidstate NMR experiments.

\section{THE AVERAGE HAMILTONIAN}

\section{A. Magnus/Floquet/Fer expansions}

The propagator expressed in the form of an infinite expansion for the three expansion schemes can be written as

$$
\begin{aligned}
U\left(\tau_{c}\right) & =\exp \left(-i \tau_{c} \sum_{n=0}^{\infty} \overline{\mathcal{H}^{(n)}}\right) \quad \text { (the Magnus expansion) } \\
& =\exp \left(-i \tau_{c} \sum_{n=0}^{\infty} \overline{\mathcal{H}_{\mathrm{Fl}}^{(n)}}\right) \quad \text { (the Floquet expansion) } \\
& =\prod_{n=0}^{\infty} \exp \left(-i \tau_{c} \overline{\mathcal{H}_{\mathrm{Fer}}^{(n)}}\right) \text {. (the Fer expansion) }
\end{aligned}
$$
by $^{1-3}$

$$
\begin{aligned}
\overline{\mathcal{H}^{(0)}}= & \frac{1}{\tau_{c}} \int_{0}^{\tau_{c}} \mathcal{H}_{0}(t) d t, \\
\overline{\mathcal{H}^{(1)}}= & \frac{-i}{2 \tau_{c}} \int_{0}^{\tau_{c}} d t_{2} \int_{0}^{t_{2}} d t_{1}\left[\mathcal{H}_{0}\left(t_{2}\right), \mathcal{H}_{0}\left(t_{1}\right)\right], \\
\overline{\mathcal{H}^{(2)}}= & \frac{-1}{6 \tau_{c}} \int_{0}^{\tau_{c}} d t_{3} \int_{0}^{t_{3}} d t_{2} \int_{0}^{t_{2}} d t_{1}\left\{\left[\mathcal{H}_{0}\left(t_{3}\right),\right.\right. \\
& {\left.\left.\left[\mathcal{H}_{0}\left(t_{2}\right), \mathcal{H}_{0}\left(t_{1}\right)\right]\right]+\left[\mathcal{H}_{0}\left(t_{1}\right),\left[\mathcal{H}_{0}\left(t_{2}\right), \mathcal{H}_{0}\left(t_{3}\right)\right]\right]\right\} . }
\end{aligned}
$$

To apply the Floquet theory, we assume that the Hamiltonian is expanded in a Fourier series of the form

$$
\mathcal{H}_{0}(t)=\sum_{n=-\infty}^{\infty} H_{n} e^{i n \omega t},
$$

where $\omega$ is the characteristic frequency and

$$
H_{n}=\frac{1}{\tau_{c}} \int_{0}^{\tau_{c}} d t \mathcal{H}_{0}(t) e^{-i n \omega t} \text { and } \omega=\frac{2 \pi}{\tau_{c}} .
$$

Note that we assumed the so-called single-mode Floquet Hamiltonian for simplicity. The average Hamiltonians can be obtained by inserting Eq. (5) into Eq. (3), and the corresponding Hamiltonians are given by ${ }^{7}$

$$
\begin{aligned}
\overline{\mathcal{H}_{\mathrm{Fl}}^{(0)}}= & H_{0}, \\
\overline{\mathcal{H}_{\mathrm{Fl}}^{(1)}}= & -\frac{1}{2} \sum_{k \neq 0} \frac{1}{k \omega}\left[H_{-k}, H_{k}\right]+\sum_{k \neq 0} \frac{1}{k \omega}\left[H_{0}, H_{k}\right], \\
\overline{\mathcal{H}_{\mathrm{Fl}}^{(2)}}= & \frac{1}{3} \sum_{k \neq 0, j \neq 0, k \neq j} \frac{1}{k j \omega^{2}}\left[H_{j},\left[H_{k-j}, H_{-k}\right]\right] \\
& +\frac{1}{2} \sum_{k \neq 0} \frac{1}{(k \omega)^{2}}\left[H_{k},\left[H_{0}, H_{-k}\right]\right]-\frac{1}{2} \sum_{k \neq 0} \frac{1}{(k \omega)^{2}}\left[H_{0},\left[H_{0}, H_{k}\right]\right] \\
& +\sum_{k \neq 0, j \neq 0} \frac{1}{k j \omega^{2}}\left[H_{j},\left[H_{-j}, H_{k}\right]\right]+\frac{1}{2} \sum_{k \neq 0, j \neq 0} \frac{1}{k j \omega^{2}}\left[H_{j},\left[H_{k}, H_{0}\right]\right] .
\end{aligned}
$$

Since these average Hamiltonians are derived directly from those using the Magnus expansion (Eq. (3)), it is natural to expect both to give identical results. In fact, we obtained the same Hamiltonians for the three test cases considered later. Hence, we shall use the notation used in Eq. (3) also for the Floquet expansion.

The Fer expansion is obtained by representing the solution of the time-dependent Schrödinger equation ${ }^{8,12,13}$

$$
\frac{d U(t)}{d t}=-i \mathcal{H} U(t)
$$

as a product form of

$$
U(t)=\exp \left\{F_{1}(t)\right\} U_{1}(t)
$$

with

$$
F_{1}(t)=-i \int_{0}^{t} \mathcal{H}\left(t^{\prime}\right) d t^{\prime}
$$

These lead to the next stage of the equation, which may be written as

$$
\frac{d U_{1}(t)}{d t}=-i \mathcal{H}_{1}(t) U_{1}(t)
$$

With iterative substitution, the $n$ th-order Hamiltonian $\mathcal{H}_{n}(t)$ is obtained as

$$
\mathcal{H}_{n}(t)=\sum_{j=1}^{\infty} \frac{(-1)^{j} j}{(j+1) !} a d_{F_{n}}^{j}\left(\mathcal{H}_{n-1}(t)\right), \quad n=1,2, \ldots
$$

where $a d_{A}$ is a linear operator whose action is written as

$$
a d_{A} B=[A, B], a d_{A}^{j} B=\left[A, a d_{A}^{j-1} B\right], \text { and } a d_{A}^{0} B=B
$$

and

$$
F_{n}(t)=-i \int_{0}^{t} \mathcal{H}_{n-1}\left(t^{\prime}\right) d t^{\prime}
$$

The (n-1)th-order average Hamiltonian is simply given from $F_{n}$ as 


$$
\overline{\mathcal{H}_{\mathrm{Fer}}^{(n-1)}}=\frac{1}{\tau_{c}} \int_{0}^{\tau_{c}} \mathcal{H}_{n-1}\left(t^{\prime}\right) d t^{\prime}=\frac{i}{\tau_{c}} F_{n}\left(\tau_{c}\right) .
$$

From these equations, it is clear that the 0th-order average Hamiltonian for the three expansions are identical, $\overline{\mathcal{H}^{(0)}}=\overline{\mathcal{H}_{\mathrm{Fl}}^{(0)}}=\overline{\mathcal{H}_{\mathrm{Fer}}^{(0)}}$.

Equation (11) shows one of the salient features of the Fer expansion, that is, the $n$ th-order Hamiltonian is written as an infinite series when $n \neq 0$. Here, we shall define the first few terms in the $n$ th-order Hamiltonian as

$$
\mathcal{H}_{n}(t)=\mathcal{H}_{n, 0}(t)+\mathcal{H}_{n, 1}(t)+\mathcal{H}_{n, 2}(t)+\cdots,
$$

with

$$
\begin{aligned}
\mathcal{H}_{n, 0}(t) & =-\frac{1}{2}\left[F_{n}(t), \mathcal{H}_{n-1}(t)\right], \\
\mathcal{H}_{n, 1}(t) & =\frac{1}{3}\left[F_{n}(t),\left[F_{n}(t), \mathcal{H}_{n-1}(t)\right]\right], \\
\mathcal{H}_{n, 2}(t) & =-\frac{1}{8}\left[F_{n}(t),\left[F_{n}(t),\left[F_{n}(t), \mathcal{H}_{n-1}(t)\right]\right]\right],
\end{aligned}
$$

For future use, we define $F_{n, j}(t)$ as

$$
F_{n, j}(t)=-i \int_{0}^{t} \mathcal{H}_{n-1, j}\left(t^{\prime}\right) d t^{\prime}
$$

and the corresponding average Hamiltonian is defined as

$$
\overline{\mathcal{H}_{\mathrm{Fer}}^{(n-1, j)}}=\frac{i}{\tau_{c}} F_{n, j}\left(\tau_{c}\right) .
$$

Note here that $\overline{\mathcal{H}_{\mathrm{Fer}}^{(n)}}$ is given by

$$
\overline{\mathcal{H}_{\mathrm{Fer}}^{(n>0)}}=\sum_{j=0}^{\infty} \overline{\mathcal{H}_{\mathrm{Fer}}^{(n>0, j)}} .
$$

\section{B. Left-running Fer expansion}

It is worthy to note that the conventional form of the Fer propagator (Eq. (2)) is not suitable ${ }^{14}$ to calculate evolution of the density matrix $\rho$ because the propagator acts to the right of the density matrix as

$$
\begin{aligned}
\rho(t)= & \exp \left(-i \tau_{c} \overline{\mathcal{H}_{\mathrm{Fer}}^{(0)}}\right) \exp \left(-i \tau_{c} \overline{\mathcal{H}_{\mathrm{Fer}}^{(1)}}\right) \cdots \rho_{0} \cdots \\
& \times \exp \left(i \tau_{c} \overline{\mathcal{H}_{\mathrm{Fer}}^{(1)}}\right) \exp \left(i \tau_{c} \overline{\mathcal{H}_{\mathrm{Fer}}^{(0)}}\right) .
\end{aligned}
$$

We shall refer the Fer propagator given in Eq. (2) to as the right-running Fer propagator. The left-running Fer propagator written as

$$
U\left(\tau_{c}\right)=\cdots \exp \left(i \tau_{c} \overline{\mathcal{H}_{\mathrm{Fer}}^{\mathrm{L}(1)}}\right) \exp \left(i \tau_{c} \overline{\mathcal{H}_{\mathrm{Fer}}^{\mathrm{L}(0)}}\right)
$$

may be obtained by starting from the time-dependent Schrödinger equation of the form ${ }^{15}$

$$
\frac{d U(t)}{d t}=-i U(t) \mathcal{H}
$$

with its first solution written as

$$
U(t)=U_{1}(t) \exp \left\{F_{1}(t)\right\}
$$

The resulting $n$ th-order Hamiltonian for the left-running Fer expansion $\left(\mathcal{H}_{n}^{\mathrm{L}}(t)\right)$ becomes

$$
\mathcal{H}_{n}^{\mathrm{L}}(t)=\sum_{j=1}^{\infty} \frac{j}{(j+1) !} a d_{F_{n}}^{j}\left(\mathcal{H}_{n-1}^{\mathrm{L}}(t)\right), \quad n=1,2, \ldots
$$

with its expanded form being related to the right-handed ones (Eq. (16)) as

$$
\begin{aligned}
& \mathcal{H}_{n, 0}^{\mathrm{L}}(t)=-\mathcal{H}_{n, 0}(t), \\
& \mathcal{H}_{n, 1}^{\mathrm{L}}(t)=\mathcal{H}_{n, 1}(t), \\
& \mathcal{H}_{n, 2}^{\mathrm{L}}(t)=-\mathcal{H}_{n, 2}(t),
\end{aligned}
$$

and

$$
\overline{\mathcal{H}_{\mathrm{Fer}}^{\mathrm{L}(0)}}=\overline{\mathcal{H}_{\mathrm{Fer}}^{(0)}}
$$

Alternatively, we apply the right-running Fer expansion to $U^{-1}$ as

$$
U^{-1}(t)=\prod_{n=0}^{\infty} \exp \left(i \tau_{c} \overline{\mathcal{H}_{\mathrm{Fer}}^{\mathrm{L}(n)}}\right)
$$

with

$$
F_{n}(t)=i \int_{0}^{t} \mathcal{H}_{n-1}^{\mathrm{L}}\left(t^{\prime}\right) d t^{\prime}
$$

and

$$
\overline{\mathcal{H}_{\mathrm{Fer}}^{\mathrm{L}(n-1)}}=\frac{-i}{\tau_{c}} F_{n}\left(\tau_{c}\right) .
$$

Note that this also leads to Eq. (25). In the following, we shall use the right-running notation for simplicity unless otherwise stated.

\section{APPLICATION TO SPECIFIC PROBLEMS}

Prior to calculation of average Hamiltonians for specific problems, we would like to discuss three key points envisaged by simple inspection of the above equations. The first key point may be realized if we compare the propagators with the 0 th and 1st-order average Hamiltonians,

$$
\begin{aligned}
U\left(\tau_{c}\right) \sim & \exp \left\{-i \tau_{c}\left(\overline{\mathcal{H}^{(0)}}+\overline{\mathcal{H}^{(1)}}\right)\right\} \\
& (\text { the Magnus/Floquet expansion) } \\
\sim & \exp \left(-i \tau_{c} \overline{\mathcal{H}_{\mathrm{Fer}}^{(0)}}\right) \exp \left(-i \tau_{c} \overline{\mathcal{H}_{\mathrm{Fer}}^{(1)}}\right) \text { (the Fer expansion) }
\end{aligned}
$$

These two are not equivalent unless $\overline{\mathcal{H}^{(0)}}=\overline{\mathcal{H}_{\mathrm{Fer}}^{(0)}}=0$ or $\left[\overline{\mathcal{H}^{(0)}}\right.$, $\left.\overline{\mathcal{H}^{(1)}}\right]=0$. In the following, we examine three representative cases for a heteronuclear two-spin $1 / 2$ system $(I$ and $S$ ); (1) $\overline{\mathcal{H}^{(0)}}=\overline{\mathcal{H}_{\text {Fer }}^{(0)}}=0$ (CW decoupling), (2) $\left[\overline{\mathcal{H}^{(0)}}, \overline{\mathcal{H}^{(1)}}\right]=0$ (CP), and (3) $\left[\overline{\mathcal{H}^{(0)}}, \overline{\mathcal{H}^{(1)}}\right] \neq 0$ (R3). The relationship among the average Hamiltonians for the third case is of particular interest.

The second key point is about the relative sizes of the average Hamiltonians in the Fer expansion. The 0th and the 1st-order Hamiltonians to be considered should be $\overline{\mathcal{H}_{\mathrm{Fer}}^{(0)}}$ and $\overline{\mathcal{H}_{\text {Fer }}^{(1,0)}}$, but which one is the 2nd-order? Is it $\overline{\mathcal{H}_{\text {Fer }}^{(1,1)}}$ or $\overline{\mathcal{H}_{\mathrm{Fer}}^{(2,0)}}$ ? How about the 3rd one? We will show in Sec. III A that it is 
not difficult to evaluate the relative sizes among the average Hamiltonians obtained by using the Fer expansion; the 2ndorder Hamiltonian is $\overline{\mathcal{H}_{\mathrm{Fer}}^{(1,1)}}$ and the 3 rd one is $\overline{\mathcal{H}_{\mathrm{Fer}}^{(1,2)}}$.

The last key point is the relation between the left-running Fer average Hamiltonian and the average Hamiltonian derived by using the Magnus/Floquet Hamiltonian. Equation (25) indicates some of the signs of the average Hamiltonians using the Magnus/Floquet expansion have to be changed if we want to consider the effect on time evolution of the density matrix in order from the 0th to higher-order corrections.

\section{A. CW decoupling: $\overline{\mathcal{H}^{(0)}}=\overline{\mathcal{H}_{\text {Fer }}^{(0)}}=0$}

Here, we examine the three expansion schemes by using heteronuclear decoupling as an example. In the doubly rotating frame of the $I$ and $S$ spins, the truncated dipolar interaction and the RF irradiation with intensity of $\omega_{1}$ along the $X$ axis of the $I$ spin for $\mathrm{CW}$ decoupling is given by

$$
\mathcal{H}=d I_{Z} S_{Z}+\omega_{1} I_{X}+\Delta \omega I_{Z},
$$

where $\Delta \omega$ denotes the off resonance, and the first term represents the dipolar interaction. The propagator $U(t)=\exp (-i \mathcal{H} t)$ is divided as

$$
\begin{aligned}
U & =U_{\mathrm{rf}} U_{0}, \\
U_{\mathrm{rf}} & =\exp \left(-i \omega_{1} I_{X} t\right), \\
U_{0} & =T \exp \left(-i \int_{0}^{t} \mathcal{H}_{0}\left(t^{\prime}\right) d t^{\prime}\right),
\end{aligned}
$$

with the relevant Hamiltonian in the interaction frame defined by $U_{\mathrm{rf}}$ being

$$
\begin{aligned}
\mathcal{H}_{0}(t) & =U_{\mathrm{rf}}^{-1}\left(d I_{Z} S_{Z}+\Delta \omega I_{X}\right) U_{\mathrm{rf}} \\
& =A\left(I_{Z} \cos \omega_{1} t+I_{Y} \sin \omega_{1} t\right)
\end{aligned}
$$

and

$$
A=\Delta \omega+d S_{Z} .
$$

The cycle time $\tau_{c}$ is defined by $U_{\mathrm{rf}}\left(\tau_{c}\right)=1$, i.e.,

$$
\tau_{c}=2 \pi / \omega_{1}
$$

In the following, we describe the calculation procedure using the Fer expansion rather closely. First, we calculate $F_{1}(t)$ as

$$
\begin{aligned}
F_{1}(t) & =-i \int_{0}^{t} \mathcal{H}_{0}\left(t^{\prime}\right) d t^{\prime} \\
& =-i \frac{A}{\omega_{1}}\left\{I_{Z} \sin \omega_{1} t-I_{Y}\left(\cos \omega_{1} t-1\right)\right\},
\end{aligned}
$$

and the 0th-order average Hamiltonian is obtained as

$$
\overline{\mathcal{H}_{\mathrm{Fer}}^{(0)}}=\frac{i F_{1}\left(\tau_{c}\right)}{\tau_{c}}=0,
$$

indicating good decoupling at the 0th-order. By using Eq. (16), we have the leading term in the 1 st-order term as

$$
\mathcal{H}_{1,0}=-\frac{1}{2}\left[F_{1}(t), \mathcal{H}_{0}(t)\right]=\frac{A^{2}}{2 \omega_{1}} I_{X}\left(1-\cos \omega_{1} t\right),
$$

and the corresponding $F_{2,0}(t)$ term thus becomes

$$
\begin{aligned}
F_{2,0}(t) & =-i \frac{A^{2}}{2 \omega_{1}} I_{X} \int_{0}^{t}\left(1-\cos \omega_{1} t^{\prime}\right) d t^{\prime} \\
& =-i \frac{A^{2}}{2 \omega_{1}} I_{X}\left(t-\frac{\sin \omega_{1} t}{\omega_{1}}\right) .
\end{aligned}
$$

The 1st-order average Hamiltonian is therefore

$$
\overline{\mathcal{H}_{\mathrm{Fer}}^{(1,0)}}=\frac{i F_{2,0}\left(\tau_{c}\right)}{\tau_{c}}=\frac{A^{2}}{2 \omega_{1}} I_{X} .
$$

Note that this does not affect the spectrum of the $S$ spin. Here, we have two "next" candidates; one is $\overline{\mathcal{H}_{\mathrm{Fer}}^{(1,1)}}$ and the other is $\overline{\mathcal{H}_{\text {Fer }}^{(2,0)}}$ as the sizes of these two terms are in the same order $\left(\sim\left(A^{3} / \omega_{1}^{2}\right)\right)$. To evaluate $\mathcal{H}_{\mathrm{Fer}}^{(2,0)}$, we write $\mathcal{H}_{2,0}(t)$ as

$$
\begin{aligned}
\mathcal{H}_{2,0}(t) & =-\frac{1}{2}\left[F_{2}(t), \mathcal{H}_{1}(t)\right] \\
& =-\frac{1}{2}\left(\left[F_{2,0}, \mathcal{H}_{1,0}\right]+\left[F_{2,0}, \mathcal{H}_{1,1}\right]+\left[F_{2,1}, \mathcal{H}_{1,0}\right]+\cdots\right) .
\end{aligned}
$$

Since $F_{2,0}(t)$ is just the result of integral of $\mathcal{H}_{1,0}(t)$, they commute. Therefore, the leading term in Eq. (41) becomes 0 . Note that this holds for the higher-order terms as well. Hence, the size of $\overline{\mathcal{H}_{\mathrm{Fer}}^{(2,0)}}$ should be smaller than $A^{3} / \omega_{1}^{2}$, and the next term we should examine is $\overline{\mathcal{H}_{\mathrm{Fer}}^{(1,1)}}$.

To calculate $\overline{\mathcal{H}_{\text {Fer }}^{(1,1)}}$, we calculate $\mathcal{H}_{1,1}(t)$ as

$$
\begin{aligned}
\mathcal{H}_{1,1}(t) & =\frac{1}{3}\left[F_{1}(t),\left[F_{1}(t), \mathcal{H}_{0}(t)\right]\right] \\
& =\frac{1}{3}\left[F_{1}(t),-2 \mathcal{H}_{1,0}(t)\right] \\
& =\frac{A^{3}}{3 \omega_{1}^{2}}\left\{I_{Y} \sin \omega_{1} t\left(\cos \omega_{1} t-1\right)+I_{Z}\left(\cos \omega_{1} t-1\right)^{2}\right\},
\end{aligned}
$$

and the corresponding $F_{2,1}(t)$ is

$$
\begin{aligned}
F_{2,1}(t)= & -i \int_{0}^{t} \mathcal{H}_{1,1}\left(t^{\prime}\right) d t^{\prime} \\
= & -i \frac{1}{3} \frac{A^{3}}{\omega_{1}^{2}}\left\{\frac{I_{Y}}{4 \omega_{1}}\left(-3+4 \cos \omega_{1} t-\cos 2 \omega_{1} t\right)\right. \\
& \left.+I_{Z}\left(\frac{3}{2} t-\frac{2 \sin \omega_{1} t}{\omega_{1}}+\frac{\sin 2 \omega_{1} t}{4 \omega_{1}}\right)\right\},
\end{aligned}
$$

and its average becomes

$$
\overline{\mathcal{H}_{\mathrm{Fer}}^{(1,1)}}=\frac{i F_{2,1}\left(\tau_{c}\right)}{\tau_{c}}=\frac{A^{3}}{2 \omega_{1}^{2}} I_{Z},
$$

which is the 2nd-order average Hamiltonian in the Fer expansion.

For further pursuit of the higher orders, we now have to compare $\mathcal{H}_{1,2}$ and the second and the third terms in $\mathcal{H}_{2,0}$ (Eq. (41)). First, we calculate $\mathcal{H}_{1,2}$ as

$$
\begin{aligned}
\mathcal{H}_{1,2} & =-\frac{1}{8}\left[F_{1},\left[F_{1},\left[F_{1}, \mathcal{H}_{0}\right]\right]\right] \\
& =-\frac{1}{8}\left[F_{1}, 3 \mathcal{H}_{1,1}\right] \\
& \vdots \\
& =-\frac{A^{4}}{4 \omega_{1}^{3}} I_{X}\left(\cos \omega_{1} t-1\right)^{2} .
\end{aligned}
$$


The average Hamiltonian thus becomes

$$
\begin{aligned}
F_{2,2} & =-i \int_{0}^{t} \mathrm{~d} t \mathcal{H}_{1,2} \\
& =-i \frac{A^{4}}{16 \omega_{1}^{4}} I_{X}\left(6 \omega_{1} t-8 \sin \omega_{1} t+\sin 2 \omega_{1} t\right)
\end{aligned}
$$

and

$$
\overline{\mathcal{H}_{\mathrm{Fer}}^{(1,2)}}=\frac{i F_{2,2}\left(\tau_{c}\right)}{\tau_{c}}=-\frac{3 A^{4}}{8 \omega_{1}^{3}} I_{X}
$$

The second and the third terms in Eq. (41) are of $A^{5}$ order, hence, the next term (the 3rd-order average Hamiltonian) to be considered is $\overline{\mathcal{H}_{\mathrm{Fer}}^{(1,2)}}$. In passing, the size of $\overline{\mathcal{H}_{\mathrm{Fer}}^{(2,1)}}$, which is derived from $\mathcal{H}_{2,1}=-\frac{1}{3}\left[F_{2}(t),\left[F_{2}(t), \mathcal{H}_{1}(t)\right]\right] \sim \frac{1}{3}\left[F_{2}(t)\right.$, $\left.\mathcal{H}_{2,0}(t)\right]$, is $\sim A^{7} / \omega_{1}^{6}$. It is clear that the $\overline{\mathcal{H}_{\mathrm{Fer}}^{(n>1)}}$ terms will emerge only as 6th-order corrections and can thus be ignored practically. To conclude, up to the 5th-order, the average Hamiltonians obtained by using the Magnus expansion are related to the 1st-order Hamiltonians by using the right-running
Fer expansion as $\overline{\mathcal{H}^{(i)}}=\overline{\mathcal{H}_{\mathrm{Fer}}^{(1, i-1)}}$ with $i=1, \ldots, 5$. In other words, for most of practical NMR experiments, we only have to consider the first two sub-propagators for the Fer expansion $U\left(\tau_{c}\right)=\exp \left(-i \tau_{c} \overline{\mathcal{H}_{\mathrm{Fer}}^{(0)}}\right) \exp \left(-i \tau_{c} \overline{\mathcal{H}_{\mathrm{Fer}}^{(1)}}\right)$ and can neglect the higher-order average Hamiltonians, $\overline{\mathcal{H}_{\mathrm{Fer}}^{(n>1)}}$.

As for the Magnus and Floquet expansions, after lengthy calculations, we confirmed that the average Hamiltonians using the two expansion schemes are identical and written as follows:

$$
\begin{aligned}
& \overline{\mathcal{H}^{(0)}}=0, \\
& \overline{\mathcal{H}^{(1)}}=\frac{A^{2}}{2 \omega_{1}} I_{X}, \\
& \overline{\mathcal{H}^{(2)}}=\frac{A^{3}}{2 \omega_{1}^{2}} I_{Z} .
\end{aligned}
$$

Note that $\overline{\mathcal{H}^{(1)}}=\overline{\mathcal{H}_{\mathrm{Fer}}^{(1,0)}}$ and $\overline{\mathcal{H}^{(2)}}=\overline{\mathcal{H}_{\mathrm{Fer}}^{(1,1)}}$. As the labor of calculation of the 3rd order for the Magnus/Floquet expansion is too heavy, we have not tried to obtain this for them. The propagators derived by using the three schemes become

$$
\begin{aligned}
U\left(\tau_{c}\right) \sim & \left.\exp \left\{-i \tau_{c} \overline{\mathcal{H}^{(1)}}+\overline{\mathcal{H}^{(2)}}+\cdots\right)\right\} \quad \text { (the Magnus/Floquet expansion) } \\
\sim & \left.\exp \left\{-i \tau_{c} \overline{\mathcal{H}_{\mathrm{Fer}}^{(1,0)}}+\overline{\mathcal{H}_{\mathrm{Fer}}^{(1,1)}}+\overline{\mathcal{H}_{\mathrm{Fer}}^{(1,2)}}+\cdots\right)\right\} \cdots \\
& (\text { the right-running Fer expansion }) \\
\sim & \cdots \exp \left\{-i \tau_{c}\left(-\overline{\mathcal{H}_{\mathrm{Fer}}^{(1,0)}}+\overline{\mathcal{H}_{\mathrm{Fer}}^{(1,1)}}-\overline{\mathcal{H}_{\mathrm{Fer}}^{(1,2)}}+\cdots\right)\right\} . \\
& (\text { the left-running Fer expansion })
\end{aligned}
$$

The propagator for the Magnus/Floquet expansion and that of the right-running Fer expansion is identical up to the 2nd order for the case of CW decoupling, which can be expected because the 0th-order Hamiltonian is $0\left(\overline{\mathcal{H}^{(0)}}=\overline{\mathcal{H}_{\mathrm{Fer}}^{(0)}}=0\right)$. However, the propagator for the left-running Fer expansion indicates that application to a density operator must carefully be done as the former two propagators were built to appreciate the effects from higher-order to lower-order.

\section{B. CP: $\left[\overline{\mathcal{H}^{(0)}}, \overline{\mathcal{H}^{(1)}}\right]=0$}

The truncated Hamiltonian for the heteronuclear dipolar coupled two spins under double RF irradiation on both the $I$ and $S$ spin at "on resonance" is given by

$$
\mathcal{H}=d I_{Z} S_{Z}+\omega_{1} I_{X}+\omega_{1} S_{X}
$$

The relevant Hamiltonian in the interaction frame defined by $U_{\mathrm{rf}}(t)=\exp \left\{-i \omega_{1}\left(I_{X}+S_{X}\right) t\right\}$ becomes

$$
\begin{aligned}
\mathcal{H}_{0}(t)= & \frac{d}{2}\left\{I_{Z} S_{Z}+I_{Y} S_{Y}+\left(I_{Y} S_{Z}+I_{Z} S_{Y}\right) \sin 2 \omega_{1} t\right. \\
& \left.+\left(I_{Z} S_{Z}-I_{Y} S_{Y}\right) \cos 2 \omega_{1} t\right\} .
\end{aligned}
$$

In this section, we simply show the calculated results as they are not controversial. The Oth-order average Hamiltonian is obtained straightforwardly as

$$
\overline{\mathcal{H}^{(0)}}=\overline{\mathcal{H}_{\text {Fer }}^{(0)}}=\frac{d}{2}\left(I_{Z} S_{Z}+I_{Y} S_{Y}\right) .
$$

After lengthy calculation, we obtain the 1st-order and the 2nd-order average Hamiltonians using the three expansion schemes as

$$
\begin{aligned}
& \overline{\mathcal{H}^{(1)}}=\overline{\mathcal{H}_{\mathrm{Fer}}^{(1,0)}}=\frac{d^{2}}{16 \omega_{1}}\left(I_{X}+S_{X}\right), \\
& \overline{\mathcal{H}^{(2)}}=\overline{\mathcal{H}_{\mathrm{Fer}}^{(1,1)}}=\frac{d^{3}}{64 \omega_{1}^{2}}\left(I_{Z} S_{Z}-I_{Y} S_{Y}\right) .
\end{aligned}
$$


The propagators for $\mathrm{CP}$ thus become

$$
\begin{aligned}
U\left(\tau_{c}\right) \sim & \exp \left[-i \tau_{c}\left\{\frac{d}{2}\left(I_{Z} S_{Z}+I_{Y} S_{Y}\right)+\frac{d^{2}}{16 \omega_{1}}\left(I_{X}+S_{X}\right)+\frac{d^{3}}{64 \omega_{1}^{2}}\left(I_{Z} S_{Z}-I_{Y} S_{Y}\right)+\cdots\right\}\right] \\
& \text { (the Magnus/Floquet expansion) } \\
\sim & \exp \left\{-i \tau_{c} \frac{d}{2}\left(I_{Z} S_{Z}+I_{Y} S_{Y}\right)\right\} \exp \left[-i \tau_{c}\left\{\frac{d^{2}}{16 \omega_{1}}\left(I_{X}+S_{X}\right)+\frac{d^{3}}{64 \omega_{1}^{2}}\left(I_{Z} S_{Z}-I_{Y} S_{Y}\right)\right\}\right] \cdots
\end{aligned}
$$

(the right-running Fer expansion)

$$
\sim \cdots \exp \left[-i \tau_{c}\left\{-\frac{d^{2}}{16 \omega_{1}}\left(I_{X}+S_{X}\right)+\frac{d^{3}}{64 \omega_{1}^{2}}\left(I_{Z} S_{Z}-I_{Y} S_{Y}\right)\right\}\right] \exp \left\{-i \tau_{c} \frac{d}{2}\left(I_{Z} S_{Z}+I_{Y} S_{Y}\right)\right\}
$$

(the left-running Fer expansion).

At first glance, the former two propagators look different. However, these two are identical up to the 2nd-order as the 0th-order Hamiltonian commutes with the 1st and 2nd-order ones,

$$
\left[I_{Z} S_{Z}+I_{Y} S_{Y}, I_{X}+S_{X}\right]=\left[I_{Z} S_{Z}+I_{Y} S_{Y}, I_{Z} S_{Z}-I_{Y} S_{Y}\right]=0 .
$$

\section{R3: $\left[\overline{\mathcal{H}^{(0)}}, \overline{\mathcal{H}^{(1)}}\right] \neq 0$}

The truncated Hamiltonian for the heteronuclear dipolar coupled two spins under on-resonance RF irradiation on the $I$ spin and MAS may be written as

$$
\mathcal{H}=d(t) I_{Z} S_{Z}+\omega_{1} I_{X} .
$$

Here, the geometric part of the heteronuclear dipolar interaction $d(t)$ is modulated by MAS, which may be written as

$$
d(t)=d \cos \omega_{1} t+d_{2} \cos 2 \omega_{1} t .
$$

Note that the MAS frequency is set equal to the intensity of decoupling to fulfill the R3 condition. For simplicity, we ignore the second $d_{2} \cos 2 \omega_{1} t$ term in the following. The relevant Hamiltonian in the interaction frame defined by $U_{\mathrm{rf}}(t)$ $=\exp \left(-i \omega_{1} I_{X} t\right)$ is

$$
\mathcal{H}_{0}(t)=A\left(I_{Z}+I_{Z} \cos 2 \omega_{1} t+I_{Y} \sin 2 \omega_{1} t\right)
$$

with $A=\frac{1}{2} d S_{Z}$.

In this section, we show the calculation process in somewhat detail as the average Hamiltonians are different for the Fer expansion and the Magnus/Floquet expansion. The average Hamiltonians for the Fer expansion are obtained as follows. We have

$$
F_{1}(t)=i \frac{A}{2 \omega_{1}}\left\{I_{Z}\left(2 \omega_{1} t+\sin 2 \omega_{1} t\right)+I_{Y}\left(1-\cos 2 \omega_{1} t\right)\right\}
$$

and the 0th-order average Hamiltonian becomes

$$
\overline{\mathcal{H}_{\mathrm{Fer}}^{(0)}}=A I_{Z},
$$

indicating reintroduction (recoupling) of the heteronuclear dipolar interaction at the R3 condition. The 1st-order term is obtained as

$$
\overline{\mathcal{H}_{\mathrm{Fer}}^{(1,0)}}=\frac{1}{\tau_{c}} \int_{0}^{\tau_{c}}\left(-\frac{1}{2}\left[F_{1}(t), \mathcal{H}_{0}(t)\right]\right) d t=\frac{1}{\tau_{c}} \int_{0}^{\tau_{c}}\left(\frac{A^{2}}{4 \omega_{1}} I_{X} 2 \omega_{1} t \sin 2 \omega_{1} t\right) d t=-\frac{A^{2}}{4 \omega_{1}} I_{X}
$$

Similarly, the 2nd-order term is obtained as

$$
\overline{\mathcal{H}_{\mathrm{Fer}}^{(1,1)}}=\frac{1}{\tau_{c}} \int_{0}^{\tau_{c}}\left(\frac{1}{3}\left[F_{1},\left[F_{1}, \mathcal{H}_{0}\right]\right]\right) d t=\frac{A^{3}}{16 \omega_{1}^{2}}\left(4 \pi I_{Y}-I_{Z}\right) .
$$

Now, we calculate the average Hamiltonians using the Floquet expansion. We would like to mention that we have also calculated the average Hamiltonians for the Magnus expansion using Eq. (3) and had identical results. The $H_{n}$ terms in (4) are given by

$$
\begin{aligned}
H_{0} & =A I_{Z}, \\
H_{ \pm 2} & =\frac{A}{2}\left(I_{Z} \mp i I_{Y}\right) .
\end{aligned}
$$

The 0th to the 2nd-order average Hamiltonians are obtained as

$$
\begin{aligned}
& \overline{\mathcal{H}_{\mathrm{Fl}}^{(0)}}=H_{0}=A I_{Z}, \\
& \overline{\mathcal{H}_{\mathrm{Fl}}^{(1)}}=-\frac{1}{2}\left(\frac{1}{2 \omega_{1}}\left[H_{-2}, H_{2}\right]+\frac{1}{-2 \omega_{1}}\left[H_{2}, H_{-2}\right]\right)+\left(\frac{1}{2 \omega_{1}}\left[H_{0}, H_{2}\right]+\frac{1}{-2 \omega_{1}}\left[H_{0}, H_{-2}\right]\right)=-\frac{A^{2}}{4 \omega_{1}} I_{X},
\end{aligned}
$$


and

$$
\begin{aligned}
\overline{\mathcal{H}_{\mathrm{Fl}}^{(2)}}= & \frac{1}{2}\left(\frac{1}{4 \omega_{1}^{2}}\left[H_{2},\left[H_{0}, H_{-2}\right]\right]+\frac{1}{4 \omega_{1}^{2}}\left[H_{-2},\left[H_{0}, H_{2}\right]\right]\right)-\frac{1}{2}\left(\frac{1}{4 \omega_{1}^{2}}\left[H_{0},\left[H_{0}, H_{2}\right]\right]+\frac{1}{4 \omega_{1}^{2}}\left[H_{0},\left[H_{0}, H_{-2}\right]\right]\right) \\
& +\left(\frac{1}{4 \omega_{1}^{2}}\left[H_{2},\left[H_{-2}, H_{2}\right]\right]+\frac{1}{4 \omega_{1}^{2}}\left[H_{-2},\left[H_{2}, H_{-2}\right]\right]\right)+\frac{1}{2}\left(\frac{1}{4 \omega_{1}^{2}}\left[H_{2},\left[H_{2}, H_{0}\right]\right]+\frac{1}{4 \omega_{1}^{2}}\left[H_{-2},\left[H_{-2}, H_{0}\right]\right]\right. \\
& \left.-\frac{1}{4 \omega_{1}^{2}}\left[H_{2},\left[H_{-2}, H_{0}\right]\right]-\frac{1}{4 \omega_{1}^{2}}\left[H_{-2},\left[H_{2}, H_{0}\right]\right]\right)=\frac{A^{3}}{32 \omega_{1}^{2}}\left(\left[I_{Z}-i I_{Y}, I_{X}\right]-\left[I_{Z}+i I_{Y}, I_{X}\right]\right)-\frac{A^{3}}{16 \omega_{1}^{2}}\left(-\left[I_{Z}, I_{X}\right]+\left[I_{Z}, I_{X}\right]\right) \\
& +\frac{A^{3}}{16 \omega_{1}^{2}}\left(-\left[I_{Z}-i I_{Y}, I_{X}\right]+\left[I_{Z}+i I_{Y}, I_{X}\right]\right)+\frac{A^{3}}{32 \omega_{1}^{2}}\left(\left[I_{Z}-i I_{Y}, I_{X}\right]-\left[I_{Z}+i I_{Y}, I_{X}\right]+\left[I_{Z}-i I_{Y}, I_{X}\right]-\left[I_{Z}+i I_{Y}, I_{X}\right]\right) \\
= & -\frac{A^{3}}{16 \omega_{1}^{2}} I_{Z} .
\end{aligned}
$$

It is thus apparent that the 2nd-order average Hamiltonian using the Fer expansion (Eq. (62)) and that using the Magnus/Floquet expansion (Eq. (66)) is different, and the corresponding propagators become

$$
\begin{aligned}
U\left(\tau_{c}\right) \sim & \exp \left\{-i \tau_{c}\left(A I_{Z}-\frac{A^{2}}{4 \omega_{1}} I_{X}-\frac{A^{3}}{16 \omega_{1}^{2}} I_{Z}\right)\right\} \\
& (\text { the Magnus/Floquet expansion) } \\
\sim & \exp \left(-i \tau_{c} A I_{Z}\right) \exp \left[-i \tau_{c}\left\{-\frac{A^{2}}{4 \omega_{1}} I_{X}\right.\right. \\
& \left.\left.+\frac{A^{3}}{16 \omega_{1}^{2}}\left(-I_{Z}+4 \pi I_{Y}\right)\right\}\right] . \\
& \text { (the right-running Fer expansion) }
\end{aligned}
$$

The different 2nd-order average Hamiltonians for the Magnus/Floquet and the Fer expansions may invoke the discrepancy found for the 1st-order Hamiltonians for the secular averaging theory (SAT) and the average Hamiltonian theory (AHT), which appears also for the case of non-zero 0th-order Hamiltonian. ${ }^{3}$ The present difference in Eqs. (67) and (68) is, however, not directly related to the discrepancy, and as shown below, the two propagators are, in fact, identical up to the 2nd order. Since the discrepancy between SAT and AHT arises by expanding its applicability to all times in SAT, the equivalence of the two expansions examined in this work is understandable as the present calculation is limited at multiples of the cycle time (stroboscopic observation). ${ }^{16}$ Equivalence between averaging theories, namely, AHT, SAT, and van Vleck transformation, was discussed in details by Llor. ${ }^{17}$ Relation between the conventional stroboscopic Hamiltonian and the van Vleck effective Hamiltonian in the framework of the Floquet Hamiltonian theory was discussed extensively by Leskes $\mathrm{et} \mathrm{al}^{7}$

The apparent difference in Eqs. (67) and (68) may be explained as follows. We adopt the following theorem about the exponential function of two operators, $X$ and $Y$, and their commutator $Z=[X, Y]$. When both $X$ and $Y$ commute with $Z$, then

$$
\exp (X+Y)=\exp (X) \exp (Y-Z / 2)
$$

This theorem cannot be applied to separate $A I_{Z}$ from $\frac{A^{2}}{4 \omega_{1}} I_{X}$ $+\frac{A^{3}}{16 \omega_{1}^{2}} I_{Z}$ in Eq. (67) as they do not commute. Here, we assume that it can be applied approximately. By putting

$$
X=-i \tau_{c} A I_{Z}
$$

and

$$
Y=i \tau_{c}\left(\frac{A^{2}}{4 \omega_{1}} I_{X}+\frac{A^{3}}{16 \omega_{1}^{2}} I_{Z}\right),
$$

we have

$$
Z=i \tau_{c}^{2} \frac{A^{3}}{4 \omega_{1}} I_{Y}
$$

Hence, we approximately have

$$
\begin{aligned}
\exp (X+Y)= & \exp \left\{-i \tau_{c}\left(A I_{Z}-\frac{A^{2}}{4 \omega_{1}} I_{X}-\frac{A^{3}}{16 \omega_{1}^{2}} I_{Z}\right)\right\} \\
\sim & \exp \left(-i \tau_{c} A I_{Z}\right) \\
& \times \exp \left\{-i \tau_{c}\left(-\frac{A^{2}}{4 \omega_{1}} I_{X}+\frac{\pi A^{3}}{4 \omega_{1}^{2}} I_{Y}-\frac{A^{3}}{16 \omega_{1}^{2}} I_{Z}\right)\right\}
\end{aligned}
$$

Indeed, the difference in the 2nd-order terms for the Magnus/Floquet expansion and the Fer expansion arises from the separation of the 0th-order average Hamiltonian in the Fer expansion.

With the left-running Fer propagator for Eq. (68), it is easy to calculate time evolution of the $S$ transverse magnetization under R3 as

$$
\begin{aligned}
\rho\left(\tau_{c}\right) & =U\left(\tau_{c}\right) S_{X} U\left(\tau_{c}\right)^{-1} \\
& =U_{1}\left(\tau_{c}\right)\left\{S_{X} \cos \left(\frac{d m}{2} \tau_{c}\right)+S_{Y} \sin \left(\frac{d m}{2} \tau_{c}\right)\right\} U_{1}^{-1}\left(\tau_{c}\right)
\end{aligned}
$$

with $U_{1}$ being the 1 st-order correction term as

$$
U_{1}\left(\tau_{c}\right)=\exp \left[-i \tau_{c}\left\{\frac{A^{2}}{4 \omega_{1}} I_{X}+\frac{A^{3}}{16 \omega_{1}^{2}}\left(-I_{Z}+4 \pi I_{Y}\right)\right\}\right]
$$

and $m= \pm 1 / 2$ being the eigenvalues of $I_{Z}$. Note that $S_{X} \cos$ $\left(\frac{d m}{2} \tau_{c}\right)+S_{Y} \sin \left(\frac{d m}{2} \tau_{c}\right)$ in Eq. (74) represents the desired and ideally recoupled $S$ resonance. It is notable that calculation of the time evolution is not straightforward when we adopt the right-running version (Eq. (68)) or the Magnus propagator. Hence, we claim that the separation of the 0th-order average Hamiltonian from the higher-order terms is one of the advantages of using the left-running Fer expansion, which in certain cases makes calculation easier.

Another advantage of the Fer expansion may be appreciated if we consider a case of $(\pi A) / \omega_{1}>1$. Note that this condition can be consistent with the convergence condition of $A / \omega_{1}<1$ for these expansions. For $(\pi A) / \omega_{1}>1$, the leading 
term in $U_{1}$ becomes the $I_{Y}$ term:

$$
U_{1}\left(\tau_{c}\right) \sim \exp \left(-i \tau_{c} \frac{\pi A^{3}}{4 \omega_{1}^{2}} I_{Y}\right),
$$

which is different from that of the 1st-order average Hamiltonian derived with the Magnus/Floquet expansion. This clearly shows that, in modification of a certain pulse sequence, the largest correction term obtained by using the Magnus expansion may not be the right one as some cross terms, which are deduced automatically in the Fer expansion, can be larger and thus must be considered first.

\section{CONCLUSIONS}

Detailed calculations are shown for three common experiments in solid-state NMR to highlight the similarities and differences among the average Hamiltonian theories with the Magnus expansion, the Floquet theory, and the Fer expansion schemes. While the first two are common in solid-state NMR field, the Fer method is a relative new comer although the mathematical formalism has been known for several decades.

We showed that, while the Magnus/Floquet expansion schemes give us a clear perspective of the size of the correction associated with the order $n$, the Fer does not. Instead, the calculation involved in the Fer expansion is much less than that in the Magnus expansion. Our calculations suggest that the Fer expansion is easier in the tabulation of higher-order terms than the other methods. There have been experiments suggested recently making use of such higher-order terms, and we believe Fer expansion method could shed more light in such a scenario. ${ }^{18}$

Since the Fer expansion employs the form of a product of sub-propagators, it appears that the Fer expansion is suitable for examination of time-dependence of the density matrix for each average Hamiltonian at different orders. It becomes, however, clear that the 1st to 5th-order average Hamiltonians, which are enough to examine an NMR experiment, are included in the 1st correction of the Fer expansion. The most salient feature of the Fer expansion is therefore appreciable only when the 0th-order average Hamiltonian is not 0 . We showed that for a $\left[\overline{\mathcal{H}^{(0)}}, \overline{\mathcal{H}^{(1)}}\right] \neq 0$ case, correction arising from the separation of $\overline{\mathcal{H}^{(0)}}$ from others would manifest itself as a second-order correction in the Fer expansion. This term can be important as the relative size of this one can be larger than the other 1st and 2nd-order terms.

It is shown that the average Hamiltonians obtained by using the Magnus expansion are similar to those by using the right-running Fer expansion. By comparing with those obtained by the left-running Fer expansion (Eq. (49)), we suggest that, when we appreciate time-evolution of a density matrix evolved under 0th to higher-order average Hamiltonian in order, the sign of the odd-order terms obtained by using the Magnus expansion should be reversed. This, however, may not be very crucial, since in some cases the eigenvalues of the spin operators involved in the odd-order terms is binary, i.e., $\pm 1 / 2$ for $\operatorname{spin}=1 / 2$. Hence, the sign of the odd-order term does not affect the result. Further, for most of the multiple pulse sequences, the sequence is constructed to be symmetric $\left(\mathcal{H}_{0}(t)=\mathcal{H}_{0}\left(\tau_{c}-t\right)\right)$ to remove the odd terms. ${ }^{2}$

${ }^{1}$ U. Haeberlen and J. S. Waugh, "Coherent averaging effects in magnetic resonance," Phys. Rev. 175, 453-467 (1968).

${ }^{2} \mathrm{U}$. Haeberlen, High Resolution NMR in Solids-Selective Averaging, Advances in Magnetic Resonance, Supplement 1 (Academic Press, New York, 1976).

${ }^{3}$ M. Mehring, High Resolution NMR Spectroscopy in Solids (Springer-Verlag, Germany, 1976).

${ }^{4} \mathrm{~W}$. Magnus, "On the exponential solution of differential equations for a linear operator," Commum. Pure Appl. Math. VII, 649-673 (1954).

${ }^{5}$ J. H. Shirley, "Solution of the Shrödinger equation with a Hamiltonian periodic in time," Phys. Rev. B 138, 979-987 (1965).

${ }^{6}$ I. Scholz, J. D. van Beek, and M. Ernst, "Operator-based Floquet theory in solid-state NMR," Solid State NMR 37, 39-59 (2010).

${ }^{7}$ M. Leskes, P. K. Madhu, and S. Vega, "Floquet theory in solid-state nuclear magnetic resonance," Prog. Nucl. Magn. Reson. Spectrosc. 57, 345-380 (2010).

${ }^{8} \mathrm{~F}$. Fer, "Résolution de l'equation matricielle $\dot{U}=p U$ par produit infini d'exponentielles matricielles," Bull. Classe Sci. Acad. Roy. Belg. 44, 818829 (1958).

${ }^{9}$ P. K. Madhu and N. D. Kurur, "Fer expansion for effective propagators and Hamiltonians in NMR," Chem. Phys. Lett. 418, 235-238 (2006).

${ }^{10}$ E. S. Mananga, "Applications of Floquet-Magnus expansion, average Hamiltonian theory and Fer expansion to study interactions in solid state NMR when irradiated with the magic-echo sequence," Solid State Nucl. Magn. Reson. 55-56, 54-62 (2013).

${ }^{11}$ T. G. Oas, R. G. Griffin, and M. H. Levitt, "Rotary resonance recoupling of dipolar interactions in solid-state nuclear magnetic resonance spectroscopy," J. Chem. Phys. 89, 692-695 (1988).

${ }^{12}$ S. Klarsfeld and J. A. Oteo, "Exponential infinite-product representations of the time-displacement operator," J. Phys. A: Math. Gen. 22, 2687-2697 (1989).

${ }^{13}$ S. Blanes, F. Casas, J. A. Oteo, and J. Ros, “The Magnus expansion and some of its applications," Phys. Rep. 470, 151-238 (2009).

${ }^{14}$ K. Kumar, "On expanding the exponential," J. Math. Phys. 6, 1928-1934 (1965).

${ }^{15} \mathrm{~F}$. Casas, J. A. Oteo, and J. Ros, "Lie algebraic approach to Fer's expansion for classical Hamiltonian systems," J. Phys. A: Math. Gen. 24, 4037-4046 (1991)

${ }^{16}$ M. M. Maricq, "Application of average Hamiltonian theory to the NMR of solids," Phys. Rev. B 25, 6622-6632 (1982).

${ }^{17}$ A. Llor, "Equivalence between dynamical averaging metjods of Schrödinger equation: Average Hamiltonian, secular averaging, and Van Vleck transformation," Chem. Phys. Lett. 199, 383-390 (1992); Erratum and Addendum, ibid. 204, 217-218 (1993).

${ }^{18} \mathrm{~A}$. Brinkmann and M. Edén, "Second order average Hamiltonian theory of symmetry-based pulse schemes in the nuclear magnetic resonance of rotating solids: Application to triple-quantum dipolar recoupling,” J. Chem. Phys. 120, 11726-11745 (2004) 